\title{
1 Elastic Modulus and Hydraulic Permeability of MDCK Monolayers
}

$4 \quad{ }^{1}$ Department of Mechanical and Aerospace Engineering, ${ }^{2}$ Department of Material Science and

5 Engineering, ${ }^{3}$ J. Crayton Pruitt Family Department of Biomedical Engineering, ${ }^{4}$ Institute for Cell

6 Engineering and Regenerative Medicine, University of Florida, Gainesville, Florida

338 MAE-B

8 Department of Mechanical and Aerospace Engineering

9 University of Florida

10 Gainesville, FL 32611

12 ph: 352-392-6438

13 fax: 352-392-6565

email: t.e.angelini@ufl.edu

ABSTRACT The critical role of cell mechanics in tissue health has led to the development of many in vitro methods that measure the elasticity of the cytoskeleton and whole cells, yet the connection between these local cell properties and bulk measurements of tissue mechanics remains unclear. To help bridge this gap, we have developed a monolayer indentation technique for measuring multi-cellular mechanics in vitro. Here, we measure the elasticity of cell monolayers and uncover the role of fluid permeability in these multicellular systems, finding that the resistance of fluid transport through cells controls their force-response at long times.

24 Keywords: Mesoscale, Cell Mechanics, Contact Mechanics, Indentation, Permeability 


\section{1. Introduction}

2 The material properties of the cytoskeleton and the cell as a whole correlate with cell

3 behavior and tissue-level physiology (Discher et al., 2005). Numerous experimental methods for

4 measuring the material properties of cells at local spatial scales exist. Measurements at sub-

5 cellular length-scales have been performed by attaching super-paramagnetic beads to the

6 cytoskeleton and applying a torque with a magnetic field (Bursac et al., 2005; Wang et al., 2002).

7 Indentations have been performed on the actin cortex using atomic-force microscopes, also at the

8 sub-cellular length-scale (Mahaffy et al., 2000; Sen et al., 2005). Whole cells have been stretched

9 between micro-cantilevers (Fernández et al., 2006) and within optical traps (Guck et al., 2005).

10 Some of these single-cell methods have been applied to cells in monolayers to gain insight into

11 tissue-level multi-cellular mechanics (Trepat et al., 2006). Within tissues, cell groups are often

12 under compression, and may exhibit collective mechanical responses different from those

13 previously determined from local shear and tensile testing methods. Thus, in vitro measurements

14 of cells under compression at the multicellular scale may reveal unexplored forces potentially at

15 play within tissues.

16 Here we apply gentle, direct contact forces to Madin Darby Canine Kidney (MDCK) cell

17 monolayers, compressing cell groups with steady forces and with no apparent cell damage. At

18 short times, we determine an elastic modulus of $33.0 \mathrm{kPa}$, which drops to $15.6 \mathrm{kPa}$ when the

19 cytoskeleton is relaxed with blebbistatin. Over long times, the cells under the indenter compress

20 slowly without translating, indicating that fluid driven out from under the contact at a rate limited

21 by the monolayer's permeability. We combine Darcy's law with a contact mechanics model for

22 thin layers to determine the monolayer permeability. These results show that while cell elasticity 
1 may dominate force-response in tissues at short times, dissipative resistance to fluid flow

2 controls tissue response at long time-scales.

4 2. Methods and Materials

$5 \quad 2.1$ Cell Culture protocols

6 MDCK cells are cultured in Dublecco's modified Eagle's media supplemented with $10 \%$

7 fetal bovine serum and $1 \%$ penicillin streptomycin at $37{ }^{\circ} \mathrm{C}$ in a $5 \% \mathrm{CO}_{2}$ atmosphere. Monolayer

8 islands, 3-5 mm in diameter, are spotted onto fibronectin coated, glass-bottomed petri dishes and

9 fluorescently dyed with 5-chloromethyl-fluorescein diacetate (CMFDA). Detailed protocols for

10 creating monolayer islands, fluorescent labelling, and several different pharmaceutical treatments

11 employed in this study can be found in the Supplementary Information.

\subsection{In situ monolayer indentation}

14 To perform tests on monolayers, we designed a micro-indentation system delicate enough to

15 deform cells without damage, using a maximum force of $50 \mu \mathrm{N}$. At this load, the contact width is

16 about $250 \mu \mathrm{m}$, so the average pressure under contact is about $1 \mathrm{kPa}$, or roughly $1 / 10$ the typical

17 modulus of epithelial tissue (Fig. 2). We are able to repeatedly apply direct pressure to

18 monolayers, hold a normal load, and retract without observing any cell damage (Fig. 2 B-D). To

19 eliminate adhesion to the cells, the indenter tip is coated in f-127 pluronic before each

20 experiment. Each of the tests described below were performed on three different monolayers.

222.3 Indentation sequence for monolayer studies 
To measure the monolayer response to contact forces, we rapidly ramp the applied load from

20 to $50 \mu \mathrm{N}$ over a $10 \mathrm{~s}$ period, hold for $600-700 \mathrm{~s}$, and remove the load by retracting the indenter.

3 We see the indentation depth rise rapidly without any apparent lag as the force is ramped to 50

$4 \mu \mathrm{N}$, then continue to rise slowly as the force is held - a behavior reminiscent of poroelastic

5 materials under applied step-loads. Previously, we observed water passing between MDCK cells

6 under low pressures over long times (Zehnder et al., 2015a, 2015c), further leading us to treat the

7 monolayers as poroelastic in the analysis described here. To characterize poroelastic materials,

8 experiments are typically split into three different regimes of mechanical response: short times,

9 where no fluid can flow and the material is incompressible, having a Poisson ratio of $v=1 / 2$; long

10 times where flow has stopped and equilibrium levels of compression are achieved; intermediate

11 times where permeability limits the rate of indentation (Hu et al., 2010). At short times, since $v$ is

12 known, $E^{*}=E /\left(1-v^{2}\right)=4 / 3 E$, where $E^{*}$ is the contact modulus and $E$ is Young's modulus.

13 This modulus is typically used again for analysis at long times to determine $v$ at equilibrium after

14 flow has stopped. Here we follow the same protocol, but we do not attempt to determine $v$

15 because the internal cell architecture may evolve over very long times. Indentation tests with

16 simultaneous imaging of the cytoskeleton are required to study the long time limit of a

17 poroelastic interpretation of mechanical data.

\section{3. Results}

\section{3.1 Thin slab contact mechanics of monolayers}

20 At the start of the indentation protocol, the contact width, $a$, grows rapidly with indentation

21 depth, $d$, due to the large disparity between the indenter radius $(1.6 \mathrm{~mm})$ and the monolayer

22 thickness $(7 \mu \mathrm{m})$. For example, $a$ exceeds the layer thickness when $d$ is just $15 \mathrm{~nm}$. Thus, the 3D

23 elasticity problem studied here falls within a thin slab limit that can be described by the Winkler 
1 elastic foundation model (Johnson, 1985). In this limit, for a spherical indenter pressing on a thin

2 sheet of thickness $h$ and contact modulus $E^{*}$, the relationship between normal force, $F_{n}$ and

3 indentation depth is given by $F_{n}=\pi E^{*} R h^{-1} d^{2}$. This relationship arises from the lateral confinement

4 of local stresses by the rigid substrate; stresses are approximately uniform along the indentation

5 direction and propagate laterally by the slab thickness, $h$. Consequently, this model will not

6 apply to tests performed on soft substrates having an elastic modulus comparable to the cell

7 layer.

8 To test whether this simple elastic slab model describes monolayer mechanics at short times,

9 we examine the scaling between the $F$ and $d$ data-points during the first 10 seconds of

10 indentation. We find that $d^{2}$ is proportional to $F$, and that data from different experiments can be

11 collapsed onto a universal scaling curve when normalized by the constant system parameters

12 (Fig. 3). For each measurement, $E^{*}$ is determined by fitting the Winkler model to the data, with

13 all other parameters fixed to their known quantities. Averaging across measurements performed

14 on different monolayers, we find $E^{*}=33.0 \pm 3.0 \mathrm{kPa}$ (mean \pm standard deviation). Cell stiffness

15 is linked to cytoskeletal pre-stress, driven by Myosin II, so to check whether the modulus

16 measured here is driven by the same underlying mechanics, we repeat the experiments on cells

17 drugged with $100 \mu \mathrm{M}$ blebbistatin, a Myosin II inhibitor. We find $E^{*}=15.6 \pm 5.5 \mathrm{kPa}$ for

18 blebbistatin treated cells, about half that of untreated cells. At short times, when no fluid can

19 flow, the effective Poisson ratio is $1 / 2$, allowing the Young's modulus, $E$, to be determined from

$20 E^{*}$. We find $E=24.8 \mathrm{kPa}$ and $11.7 \mathrm{kPa}$ for untreated and blebbistatin treated monolayers, 21 respectively. 
In poroelastic materials, fluid permeability controls the response to applied pressure over

2 intermediate time-scales. Our measurements reveal that this time-scale for cell monolayers is on

3 the order of hundreds of seconds for pressures in the kilopascal range. By feeding back on the

4 capacitance probe signal, our instrument can hold a constant force of $50 \mu \mathrm{N}$ for these long

5 durations (Fig 4A). A remaining challenge, however, is the measurement of indentation depth

6 over these time-scales during which we expect fluid to flow through the monolayer. Small

7 variations in temperature that result in uncontrolled length changes of mechanical components

8 make it impossible to determine micron-scale changes in indentation depth from the piezo stage

9 position, which is moving to hold a constant force. We therefore determine the indentation depth

10 by analyzing images of the monolayer, measuring the area of contact between the monolayer and

11 the hemisphere. The contact area, $A$, is measured with image processing code that identifies the

12 edge of deformed regions, and we infer an average contact radius, $a$, from $A=\pi a^{2}$. For a sphere

13 pressing into a flat surface, the contact radius can be used to determine a maximum indentation

14 depth, $d$, at the center of the indent from $a=\sqrt{2 R d}$, where $R=1.6 \mathrm{~mm}$ is the radius of curvature

15 of the hemispherical indenter.

16 To determine the cell layer permeability at intermediate times, we employ the surface

17 pressure distribution from the Winkler model, given by $P(r, t)=\frac{E^{*}}{2 R h}\left(a^{2}(t)-r^{2}\right)$, where $r$ is

18 the radial distance from the center of contact. The resistance to flow of fluid out of the region

19 directly under indentation depends on the permeability of the system, which can be defined

20 generally by Darcy's law, which can be written in many different forms. Here, we use a form of

21 Darcy's law that relates the local velocity, $\mathbf{v}(\mathbf{r}, t)$, of fluid passing through a porous material of

22 thickness, $h$, with a permeability $k_{e f f}$, due to a local pressure gradient $\nabla P(\mathbf{r}, t)$, given by $\mathbf{v}(\mathbf{r}, t)=$

$23 \frac{k_{e f f}}{h} \nabla P(\mathbf{r}, t)$. Using the Winkler pressure distribution in cylindrical coordinates, the gradient is 
$1 \quad \nabla P(r, \mathrm{t})=\frac{-E^{*}}{R h} r \hat{r}$. The velocity of the fluid at the edge of contact, moving outward at a flow rate

$2 Q$ is given by $\mathbf{v}(a, t)=\frac{Q(t)}{2 \pi a(t) h} \hat{r}$, where the denominator is the surface area of a cylindrical shell

3 below the edge of contact. Equating these velocities at the boundary, $r=a$, we solve for the

4 permeability, finding $k_{e f f}=\frac{R h}{2 E^{*}} \cdot \frac{Q(t)}{\pi a^{2}}$. We employ volume conservation to write the volumetric

5 flow rate as $Q(t)=\pi \cdot 2 R d \dot{d}$, where $\dot{d}$ is the time derivative of the indentation depth. We use

6 this relationship to eliminate $Q$ in the permeability formula, finding $k_{e f f}=\frac{R h}{2 E^{*}} \cdot \dot{d}$. A linear fit of

7 the data (Fig. 4C) is used to determine $\dot{d}$, yielding $k_{e f f}=0.22 \pm 0.01 \mu \mathrm{m}^{3} \mathrm{kPa}^{-1} \mathrm{~s}^{-1}$ for undrugged

8 cells, and $k_{\text {eff }}=0.31 \pm 0.03 \mu \mathrm{m}^{3} \mathrm{kPa}^{-1} \mathrm{~s}^{-1}$ for blebbistatin treated cells.

9 In the MDCK monolayers studied here, cells are connected to one another through fluid-

10 permeable gap junctions, which allow water, ions, and small molecules to pass between cells and

11 are likely the limiting factor for inter-cellular fluid flow (Zehnder et al., 2015a, 2015b, 2015c).

12 The potential mechanical coupling between cell contractility, cell morphology, and gap-junction

13 stability may explain this modest increase in the permeability of monolayers with inhibited

14 cytoskeletal contraction, though further investigation is required to verify such an underlying

15 mechanism. It is also intriguing that, while the blebbistatin-treated monolayer is slightly more

16 permeable, the indentation rate is decreased (Fig. 4B). We interpret this observation by

17 considering that $\dot{d}$ is linearly proportional to $E^{*}$, arising from the proportionality between $\nabla P$ and

$18 E^{*}$ in the Winkler model. Thus, a lower modulus may serve as a mechanism to reduce persistent

19 fluid flow in monolayers with increased permeability. We perform control studies to test the

20 potential role of inter-cellular gap junction permeability. We block gap junctions by treating

21 monolayers with Carbenoxolone ( $\mathrm{CBX})$, finding that large-amplitude undulations emerge at the

22 monolayer apical surface (Fig. 4C). This surface roughness prohibits the interpretation of 
1 indentation experiments with the model developed here, so we perform a qualitative test on un-

2 drugged individual cells. These cells rupture at applied loads of $50 \mu \mathrm{N}$ or less, suggesting that

3 gap-junction permeability may be essential to allowing volume change under applied mechanical

4 pressure (see Supplementary Information for experimental details). Finally, we perform a control

5 experiment that tests the role of water exchange with the liquid bath across the cell membrane.

6 Indentation tests on cells treated with the chloride channel blocker 5-nitro-2-(3-

7 phenylpropylamino)-benzoic acid (NPPB) reveal a modulus and permeability comparable to that

8 of un-drugged cells (see Supplementary Information and Fig. 4C).

\section{4. Discussion}

11 The instrument and experimental approach developed here represent a bridge between 12 traditional mechanical techniques, in which macroscopic slabs of tissue are stretched, sheared, or

13 indented, and more recently developed techniques that probe individual cells, one at a time.

14 Similar to the way that traction force microscopy was extended to the meso-scale to study multi-

15 cellular forces (Tambe et al., 2011; Trepat et al., 2009), our meso-scale indentation method

16 allows in vitro systems like monolayers to be probed as multi-cellular model tissues with the

17 level of control needed to elucidate the basic principles of collective cell mechanics. In future

18 work, as instrument sensitivity and in situ imaging improves, more advanced questions about cell

19 monolayer contact mechanics may be explored (Ateshian, 2009; Dapp et al., 2014; Nalam et al., 20 2015).

21 In recent work, the elastic moduli of single $3 \mathrm{~T} 3$ fibroblasts and of small islands of 22 keratinocytes have been inferred by combining traction-force microscopy with continuum

23 theoretical models (Mertz et al., 2012; Oakes et al., 2014), finding moduli about an order of 
1 magnitude lower than measured here. This discrepancy may reflect variation in mechanics

2 between cell types, but may also arise from the need to perform traction force measurements on

3 soft substrates, which softens the cells. Direct measurements of single MDCK cells with

4 microrheological techniques also reveal a lower modulus than measured here (Balland et al.,

5 2006), while the elastic modulus of MDCK monolayers on collagen scaffolds agree with our

6 results (Harris et al., 2012). Thus, it will be interesting in the future to investigate how cells may

7 stiffen upon culturing in monolayer. Here we have found a potential contributor to this difference

8 between monolayer and single cell mechanics; the notion that fluid transport contributes to

9 multi-cellular mechanics has been inferred from passive observation, but measurements of the

10 associated forces have not been performed previously (Zehnder et al., 2015c). The permeability

11 measured here differs by less than an order of magnitude from this previous prediction, and

12 further indicates that the forces associated with intercellular fluid flow play a significant role in

13 multicellular mechanics within tissues, in addition to the well-established elastic forces of the 14 cytoskeleton.

\section{Sources of funding}

19 This project was funded by National Science Foundation grant No. CMMI-1161967. 


\section{References}

2 Ateshian, G.A., 2009. The role of interstitial fluid pressurization in articular cartilage lubrication. J. Biomech. doi:10.1016/j.jbiomech.2009.04.040

Balland, M., Desprat, N., Icard, D., Fereol, S., Asnacios, A., Browaeys, J., Henon, S., Gallet, F., 2006. Power laws in microrheology experiments on living cells: Comparative analysis and modeling. Phys. Rev. E 74, 21911. doi:10.1103/PhysRevE.74.021911

Bursac, P., Fabry, B., Lenormand, G., Oliver, M., Weitz, D.A., Viasnoff, V., Butler, J.P., Fredberg, J.J., 2005. Cytoskeletal remodelling and slow dynamics in the living cell. Nat. Mater. 4, 557-61. doi:10.1038/nmat1404

Dapp, W.B., Prodanov, N., Müser, M.H., 2014. Systematic analysis of Persson's contact mechanics theory of randomly rough elastic surfaces. J. Phys. Condens. Matter 26, 355002. doi:10.1088/0953-8984/26/35/355002

Discher, D.E., Janmey, P., Wang, Y.-L., 2005. Tissue cells feel and respond to the stiffness of their substrate. Science 310, $1139-43$. doi:10.1126/science.1116995

Fernández, P., Pullarkat, P. a, Ott, A., 2006. A master relation defines the nonlinear viscoelasticity of single fibroblasts. Biophys. J. 90, 37963805. doi:10.1529/biophysj.105.072215

Guck, J., Schinkinger, S., Lincoln, B., Wottawah, F., Ebert, S., Romeyke, M., Lenz, D., Erickson, H.M., Ananthakrishnan, R., Mitchell, D., Kas, J., Ulvick, S., Bilby, C., 2005. Optical deformability as an inherent cell marker for testing malignant transformation and metastatic competence. Biophys J 88, 3689-3698. doi:10.1529/biophysj.104.045476

Harris, A.R., Peter, L., Bellis, J., Baum, B., Kabla, A.J., Charras, G.T., 2012. Characterizing the mechanics of cultured cell monolayers. Proc. Natl. Acad. Sci. U. S. A. 109, 16449-54. doi:10.1073/pnas.1213301109

Hu, Y., Zhao, X., Vlassak, J.J., Suo, Z., 2010. Using indentation to characterize the poroelasticity of gels. Appl. Phys. Lett. 96, 1-4. doi:10.1063/1.3370354

Johnson, K.L., 1985. Contact mechanics. Cambridge University Press, Cambridge, pp. 104-106.

22 Mahaffy, R.E., Shih, C.K., MacKintosh, F.C., Kas, J., 2000. Scanning proble-based frequency-dependent microrheology of polymer gels and biological cells. Phys. Rev. Lett. 85, 880-883.

24 Mertz, A.F., Banerjee, S., Che, Y., German, G.K., Xu, Y., Hyland, C., Marchetti, M.C., Horsley, V., Dufresne, E.R., 2012. Scaling of traction forces with the size of cohesive cell colonies. Phys. Rev. Lett. 108. doi:10.1103/PhysRevLett.108.198101

Nalam, P.C., Gosvami, N.N., Caporizzo, M. a., Composto, R.J., Carpick, R.W., 2015. Nano-rheology of hydrogels using direct drive force modulation atomic force microscopy. Soft Matter 11, 1-3. doi:10.1039/C5SM01143D

Oakes, P.W., Banerjee, S., Marchetti, M.C., Gardel, M.L., 2014. Geometry regulates traction stresses in adherent cells. Biophys. J. 107, 825-833. doi:10.1016/j.bpj.2014.06.045

Sen, S., Subramanian, S., Discher, D.E., 2005. Indentation and adhesive probing of a cell membrane with AFM: theoretical model and experiments. Biophys. J. 89, 3203-13. doi:10.1529/biophysj.105.063826

Tambe, D.T., Hardin, C.C., Angelini, T.E., Rajendran, K., Park, C.Y., Serra-Picamal, X., Zhou, E.H., Zaman, M.H., Butler, J.P., Weitz, D.A., Fredberg, J.J., Trepat, X., 2011. Collective cell guidance by cooperative intercellular forces. Nat. Mater. 10, 469-75. doi:10.1038/nmat3025 
Trepat, X., Puig, F., Gavara, N., Fredberg, J.J., Farre, R., Navajas, D., 2006. Effect of stretch on structural integrity and micromechanics of human alveolar epithelial cell monolayers exposed to thrombin. Am. J. Physiol. Lung Cell. Mol. Physiol. 290, L1104-10. doi:10.1152/ajplung.00436.2005

Trepat, X., Wasserman, M.R., Angelini, T.E., Millet, E., Weitz, D.A., Butler, J.P., Fredberg, J.J., 2009. Physical forces during collective cell migration. Nat. Phys. 5, 426-430. doi:10.1038/nphys1269

Wang, N., Tolić-Nørrelykke, I.M., Chen, J., Mijailovich, S.M., Butler, J.P., Fredberg, J.J., Stamenović, D., 2002. Cell prestress. I. Stiffness and prestress are closely associated in adherent contractile cells. Am. J. Physiol. Cell Physiol. 282, C606-C616. doi:10.1152/ajpcell.00269.2001

Zehnder, S.M., Suaris, M., Bellaire, M.M., Angelini, T.E., 2015a. Cell volume fluctuations in MDCK monolayers. Biophys. J. 108, $247-250$. doi:10.1016/j.bpj.2014.11.1856

11 Zehnder, S.M., Wiatt, M.K., Uruena, J.M., Dunn, A.C., Sawyer, W.G., Angelini, T.E., 2015b. Multicellular density fluctuations in epithelial monolayers. Phys. Rev. E - Stat. Nonlinear, Soft Matter Phys. 92. doi:10.1103/PhysRevE.92.032729

13 Zehnder, S.M., Zegers, F.M., Angelini, T.E., 2015c. A Langevin model of physical forces in cell volume fluctuations. J. Biomech. 
1 Figure 1 (A) A micro-indenter is positioned over an inverted microscope and translated vertically

2 with a piezo driven stage. Deflection of the double-leaf flexure is measured with a capacitive

3 linear displacement sensor (3 nm resolution). A constant load is held with closed-loop feedback.

4 (B) The $1.6 \mathrm{~mm}$ radius indenter presses into the $7 \mu \mathrm{m}$ thick cell layer, applying a normal force,

$5 F_{n}$, making a contact diameter $2 a$. The true disparity between the indenter radius and the

6 monolayer thickness is much greater than illustrated here.

8 Figure 2 (A) MDCK monolayer under persistent $50 \mu \mathrm{N}$ load. Scale bar: $200 \mu \mathrm{m}$. Monolayer 9 indentation viewed at higher magnification, before (B), during (C), and after indentation (D) is 10 performed without apparent cell damage. Scale bar: $50 \mu \mathrm{m}$.

12 Figure 3 (A) Representative loading curve during the first 10s of indentation, plotting force, $F$, 13 versus the indentation depth, $d$, for an untreated monolayer. The red line is a fit of the Winkler 14 contact model to the data, where the contact modulus, $E^{*}$, is the fitting parameter. (B) Collapse of 15 four different indentation data-sets of both untreated and blebbistatin treated MDCK monolayers.

16 The datasets are collapsed by non-dimensionalizing the Winkler model $F$ - $d$ curve after fitting 17 each dataset. The collapsed data scales like $d^{2}$, as predicted by the Winkler Model.

19 Figure 4 (A) Force versus time curve of a typical indentation experiment at $50 \mu \mathrm{N}$ normal load 20 held for 600 s. (Inset) Average force fluctuations about the $50 \mu \mathrm{N}$ persistent load are less than \pm $211 \mu \mathrm{N}$ for these experiments. (B) Indentation depth versus time for the indentation experiments. 22 Following a rapid elastic deformation, the indentation depth persistently and slowly increases 
1 over the course of hundreds of seconds, consistent with poroelastic dynamics. The dashed lines

2 demarcate the saturation point of the elastic regime and the transition into poroelastic behavior.

3 (Inset) Representative contact area observations at the beginning of indentation (orange) and end

4 of indentation (yellow) for the experiments, $A_{1}=37,000 \mu \mathrm{m}^{2}$ and $A_{2}=60,000 \mu \mathrm{m}^{2}$. Contact area

5 increasing over time under persistent load is observed in all tested conditions. (C). (Left)

6 Confocal microscopy X-Z scans of MDCK monolayers before and after administration of the

7 gap junction blocker, CBX (60X magnification). The monolayer surface changes from a flat

8 (top) to rough within one hour after CBX drugging (bottom), preventing analysis using the

9 Winkler model. (Right) The average contact modulus and permeability of monolayers under 10 different testing conditions. 


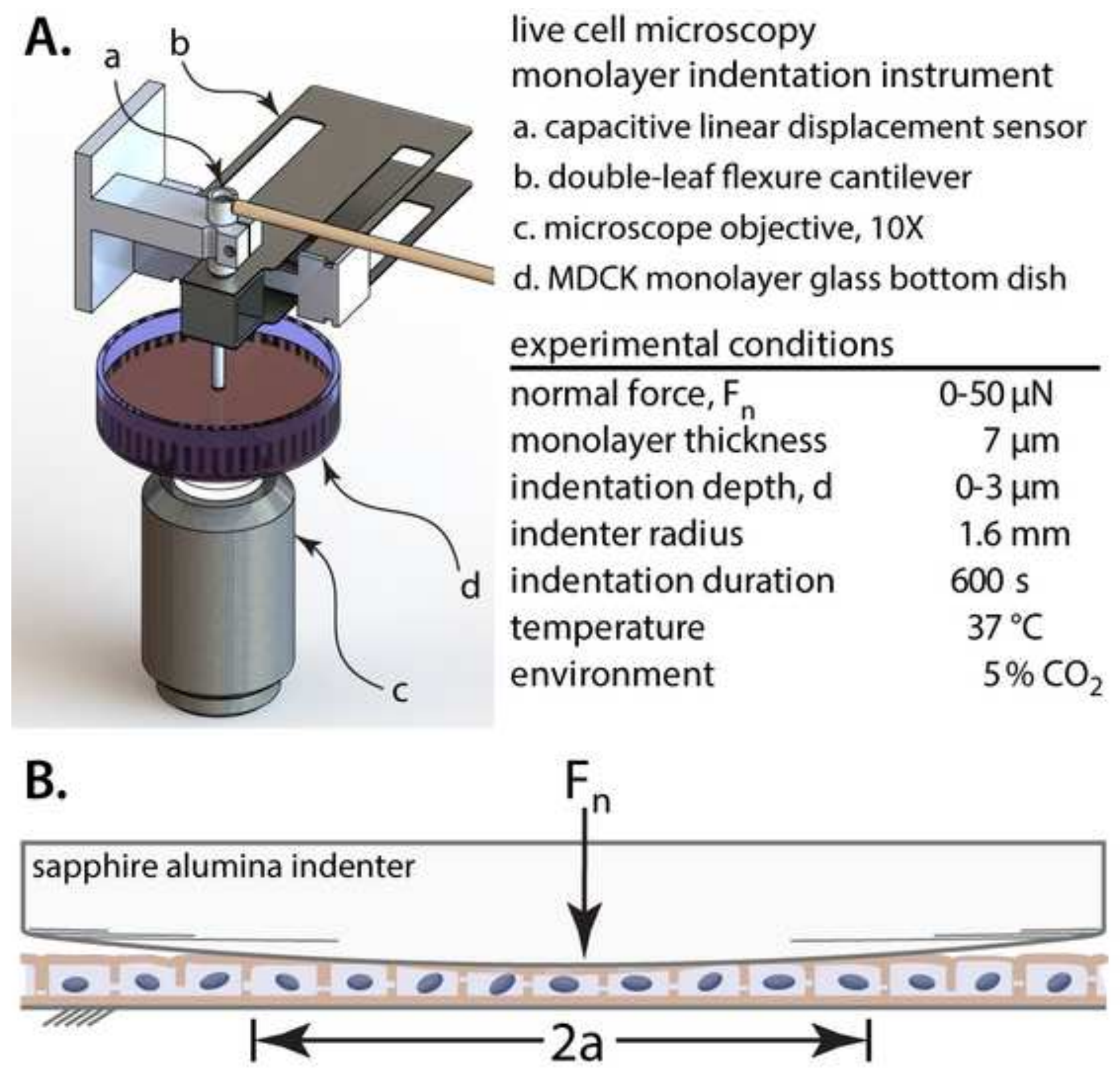



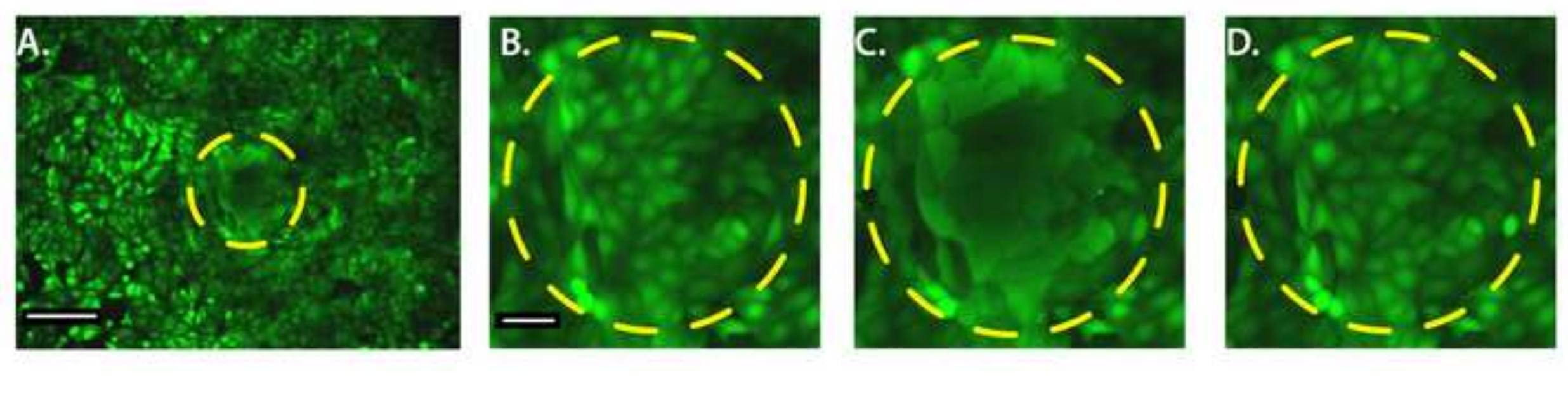

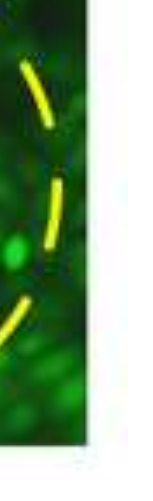
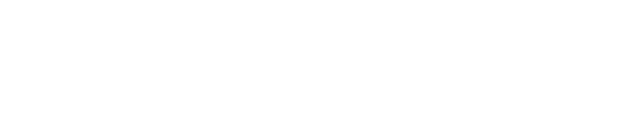
A.

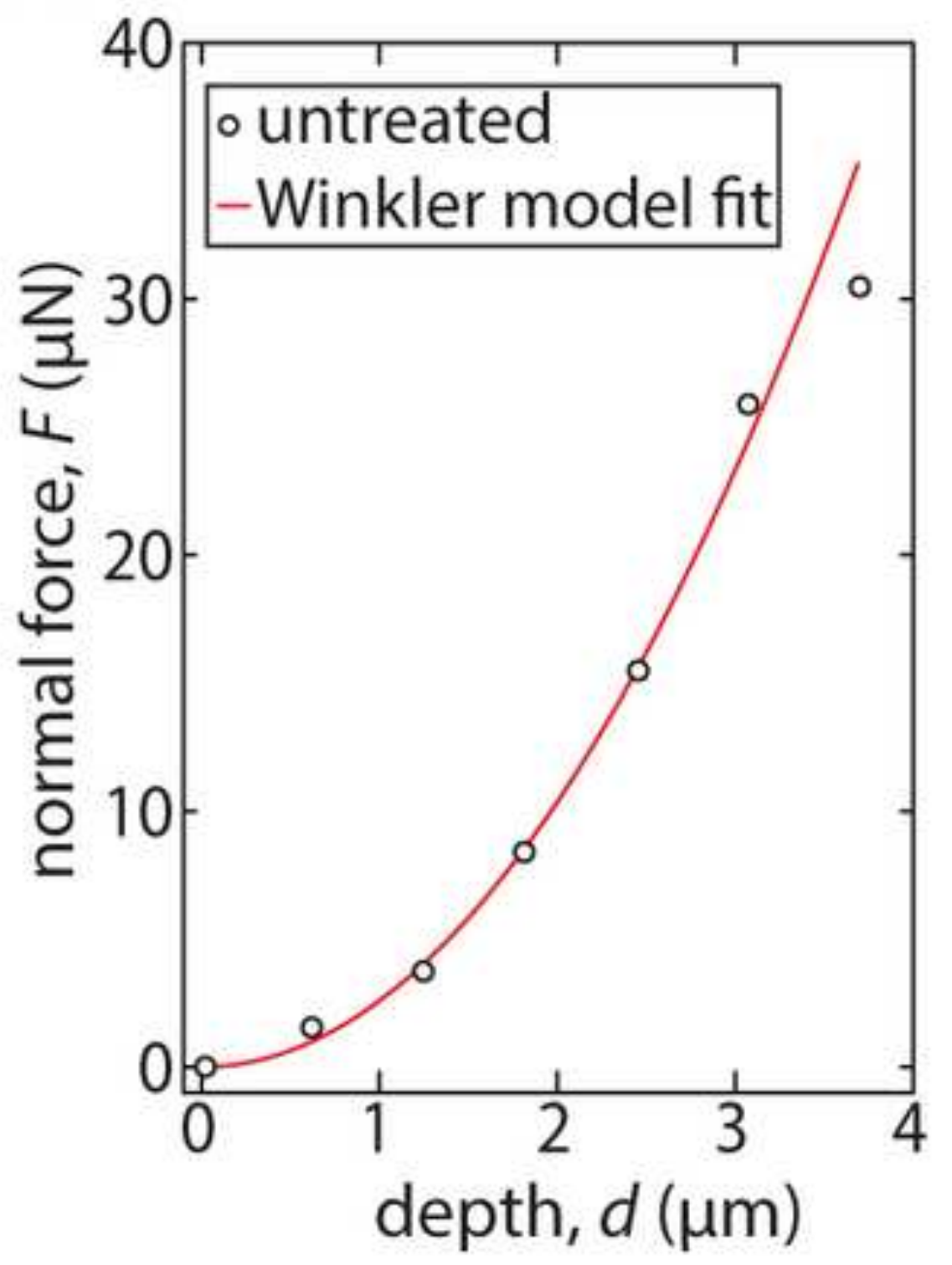

B.

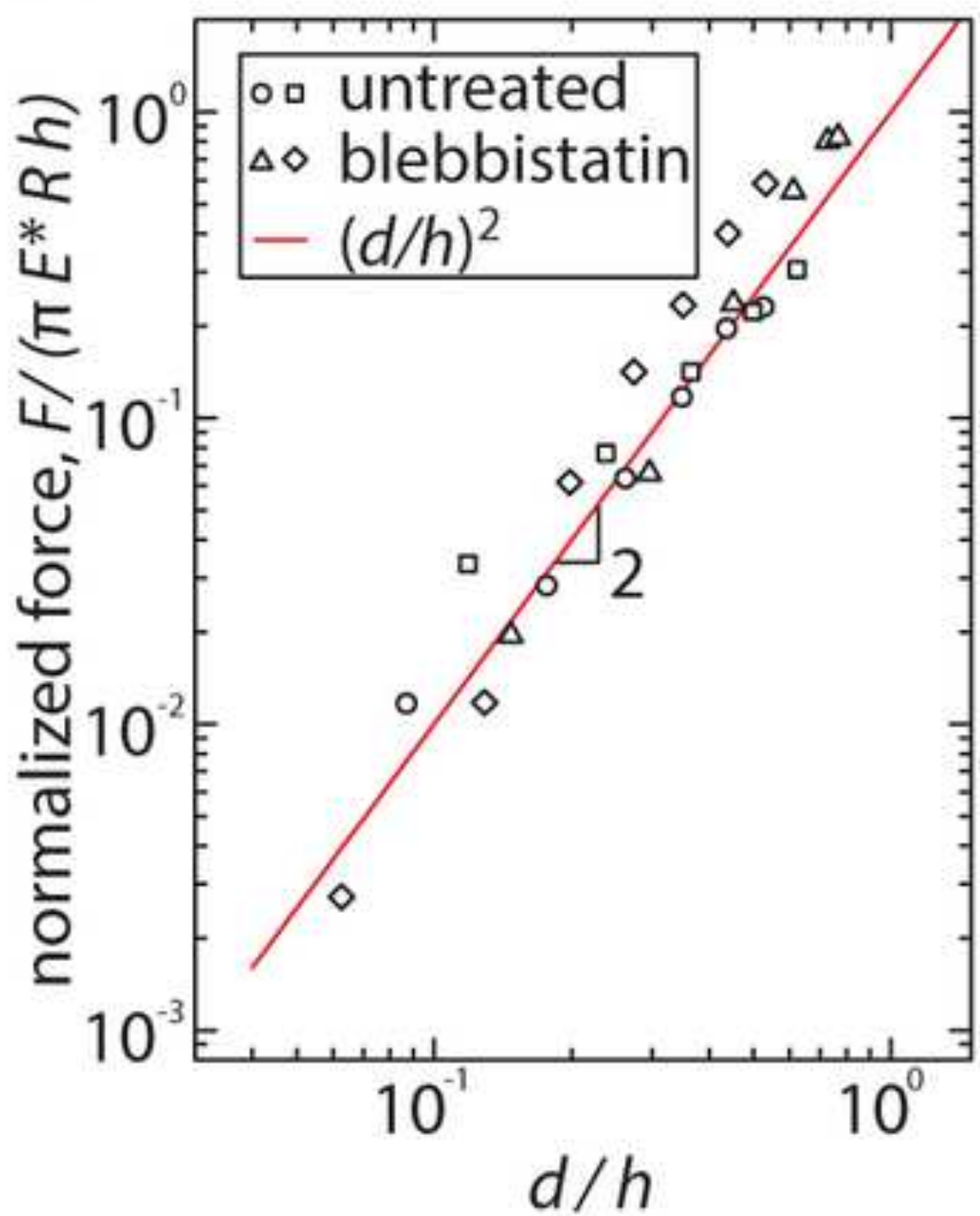



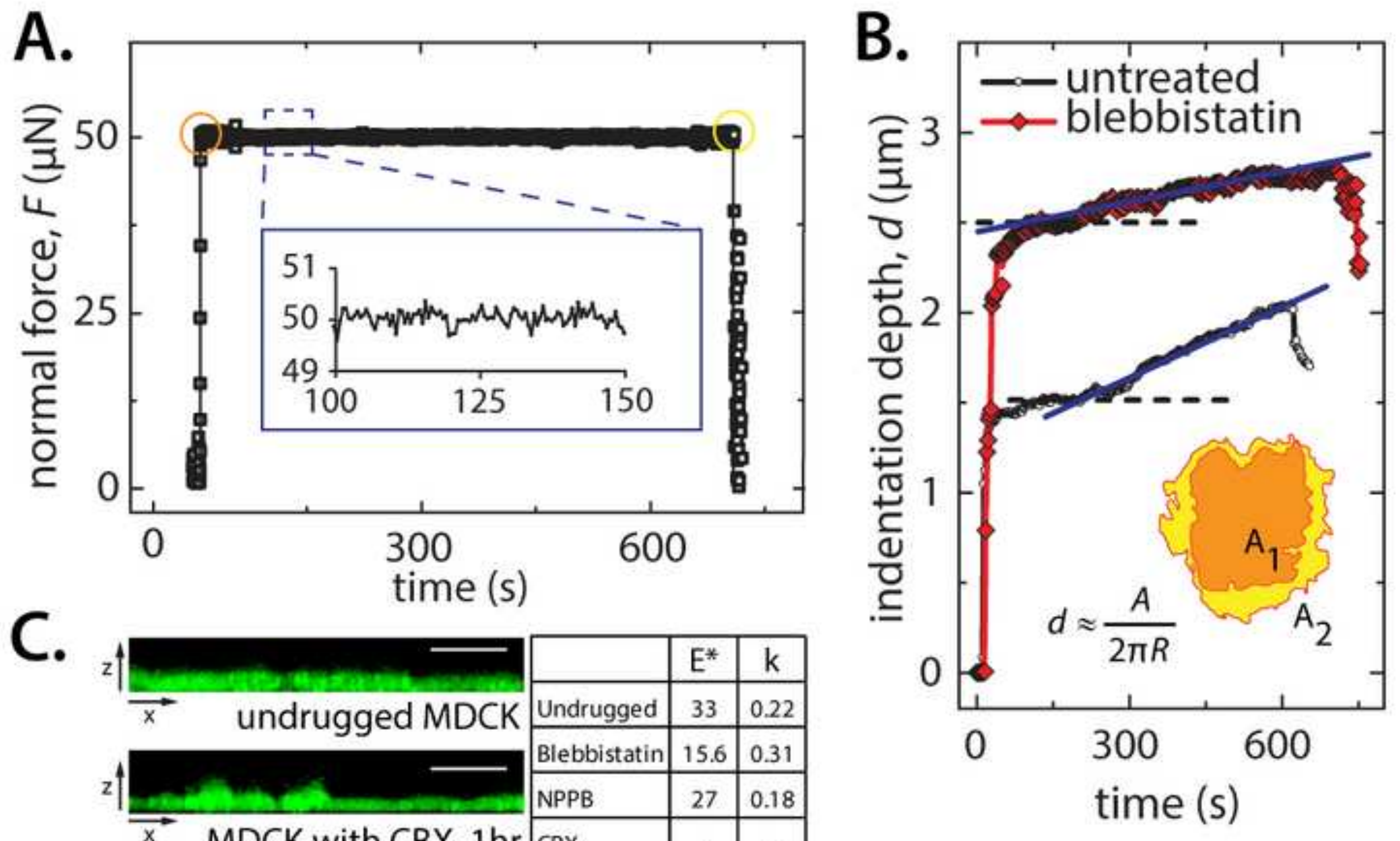